\title{
Determinants of Passenger Transport Modal Choice- A Case of Harare to Gaborone Route
}

\author{
Ephraim Govere* David Chikwre Tendai Silivaziso Mandere King Christopher Muchenje \\ Shackrod Munuhwa \\ Business Management Department, BAISAGO University, Bag 149, Gaborone, Botswana
}

\begin{abstract}
The study sought to find out what determines passenger choice of transport in cross boarder mobility looking specifically at socio-economic and travel characteristics. This was achieved with the use of methodological triangulation approach not only involving qualitative and quantitative methodology but other sources of data collection such as questionnaire administration and in-depth interviews. A sample of 30 respondents travelling from Harare to Gaborone were considered and selected from buses, private vehicles, train and air transport in Harare. The analysis began with the socio-economic and travel characteristics of respondents as to indicate which of these socio-economic and travel characteristics predominates in the study area. More so, the results of socioeconomic, travel characteristics and reasons were cross tabulated with modal choice on the route. The significant relationship between respondents' socio-economic, travel characteristics and reasons with modal choice was ascertained by using Pearson's chi-square test. The results of the study revealed that there are three modes of transport available for passengers from Harare to Gaborone route which are road, rail and air. The most preferred mode of transport amongst them is road, followed by rail and then air. Major determinants of modal choice are, speed, cost, travel time, comfort, safety, reliability and the customer service given by the operator.
\end{abstract}

Keywords: modal choice, cross border, passengers, route choice, Harare, Gaborone, transportation mode

DOI: $10.7176 / \mathrm{JESD} / 11-12-01$

Publication date:June 30th 2020

\section{Introduction}

Cross boarder long-distance travel has rapidly increased in recent decades. Technological innovation, car ownership and economic growth, globalisation, regional integration and liberalisation between nations are the major factors behind the rising demand for inter-city mobility. Improvements in highway, train and airport infrastructure have reduced travel costs and times while increasing safety. Schafer (1998) noted that travel time budgets have remained relatively constant, thus allowing people to travel further. Economic growth has also led to higher average disposable income; this favourable economic environment, along with less expensive car ownership, has contributed to the increase in long-distance business and personal trips. Increased mobility implies economic, social and environmental consequences. Differences in economic performances of different countries has also led to the increase in cross boarder mobility as seen in the increase of passengers who travel from Harare to Gaborone. According to Limtanakool et al. (2006), it permits a higher integration among regions, provides better accessibility to public services and social networks and extends the potential market for tourism activities. However, it can also have a negative environment effect, as longer trips involve increased energy consumption and greater emissions of pollutants. Furthermore, the investments required for enhancing long distance travel are substantial, implying high opportunity costs to the economy. In this sense, a deeper understanding of travel behaviour in long-distance travel may ease transport policy challenges such as making mobility more sustainable and short- and long-term individual decisions. Long term travel planning typically involves making decisions concerning car ownership and residential or work locations. The final outcome of these decisions impact trip behaviour, affecting short-term aspects such as modal choice, departure time and choice of route (Ben-Akiva and Bierlaire, 1999). To date, transportation and geographic researchers have typically studied the impact of these factors on daily and short-distance trips. Nonetheless, factors determining daily travel decisions and their interdependencies may have different impacts on less frequent events such as medium- and long-distance trips (Limtanakool et al., 2006).

Mode choice is partly determined by transport time and the relative costs of the various modes available for a specific tour or route. The importance of travel time relative to cost, i.e. the value-of-time, increases with income. Therefore, as incomes rise, people have a stronger preference for faster modes of transport (car, high-speed trains, air). Increases in income also lead to an increased desire for comfort during the tour, as well as the ability to afford more comfortable modes. As a result, increases in income tend to lead to further shifts toward automobiles and, to a lesser extent, to train and away from non-motorized modes. For long-distance interregional transport (above 500 $\mathrm{km}$ ), higher incomes shift travel from car and coach transport to high-speed train and air transport, especially in the developed world. In developed countries, increases in per capita incomes lead to a shift of modes from nonmotorized travel toward cars. (Ralph, 2011). For crossboader trips modal choice is mostly influenced by the number of trips in a specific period and purpose of such trips. Note has been taken that when one travels frequently 
such as crossboader traders the choice of transport will be cheap non-luxurious transport or economy class train. The reason being the need to make more saving by avoiding luxurious modes of transport which are expensive. However, when a passenger is less frequent mode choice becomes air, speed trains or luxuries coaches with the highest levels of passenger safety. Some long distance and crossboader passengers and travellers prefer using own private cars when the road infrastructure is ok and when there are no much regulations and administrative restriction and boarder entry into another country (Eboli \& Mazzulla, 2018).

The important socio-demographic/economic variables that influence travel behaviour include age, gender, household composition, income, and gender and car ownership, level of education, occupational status, ability to drive, person travelling with and purpose of travel. (Abane 2011; Gonzalez \& Suarez, 2013). Cross boarder passenger transport choice is derived from a complex process involving objective and subjective determinants stemming from different disciplines and interrelated to a larger or smaller extent (Gonzalez \& Suarez, 2013). Modal choice is defined as the decision process to choose between different transport alternatives, which is determined by a combination of individual socioeconomic, travel and spatial characteristics, and influenced by socio-psychological factors. Abane (2011) and Buehler (2011) have conducted studies on modal choice using different number of factors. These studies do not make a clear distinction between short and long distance studies. Long distance trips are masked in threshold standard that are intercity, interurban or interregional by design, (Ralph, 2011).

Specific objectives are to:

1. Find out the socio-economic, travel characteristics and reasons for modal choice on Harare to Gaborone cross boarder route;

2. Analyse the relationship between socio-economic travel characteristics and modal choice

3. Assess how individuals perceive the modal choices on the route

\section{Literature review}

\subsection{Transport modes}

Transport modes are the means by which passengers and freight achieve mobility. They are mobile transport assets and fall into three basic types; land (road, rail and pipelines), water (shipping), and air transport modes are designed to either carry passengers or freight, but most modes can carry a combination of both. Each mode is characterized by a set of technical, operational and commercial characteristics. Technical characteristics relate to attributes such as speed, capacity, and motive technology while operational characteristics involve the context in which modes operated, including speed limits, safety conditions or operating hours, (Rodrigue ,2020). Although intermodal transportation has opened many opportunities for complementarity between modes, transport operators are now competing over many modes in the transport chain.

Rodrigue, (2020) further alluded that modal usage is an important aspect which relates to competition that involves comparative advantage of using a specific or a combination of modes. Distance remains one of the basic determinants of modal usage for passenger transportation. However, for a similar distance, costs, speed, and comfort can be significant factors behind the choice of a mode or sub mode. Infrastructure usage. Competition resulting from the presence of passenger traffic on the same itineraries linking the same nodes. Each level of capacity used by a mode is therefore at the expense of the other mode.

\subsection{Theoretical framework}

2.2.1 Modal choice model

Figure: 2.1

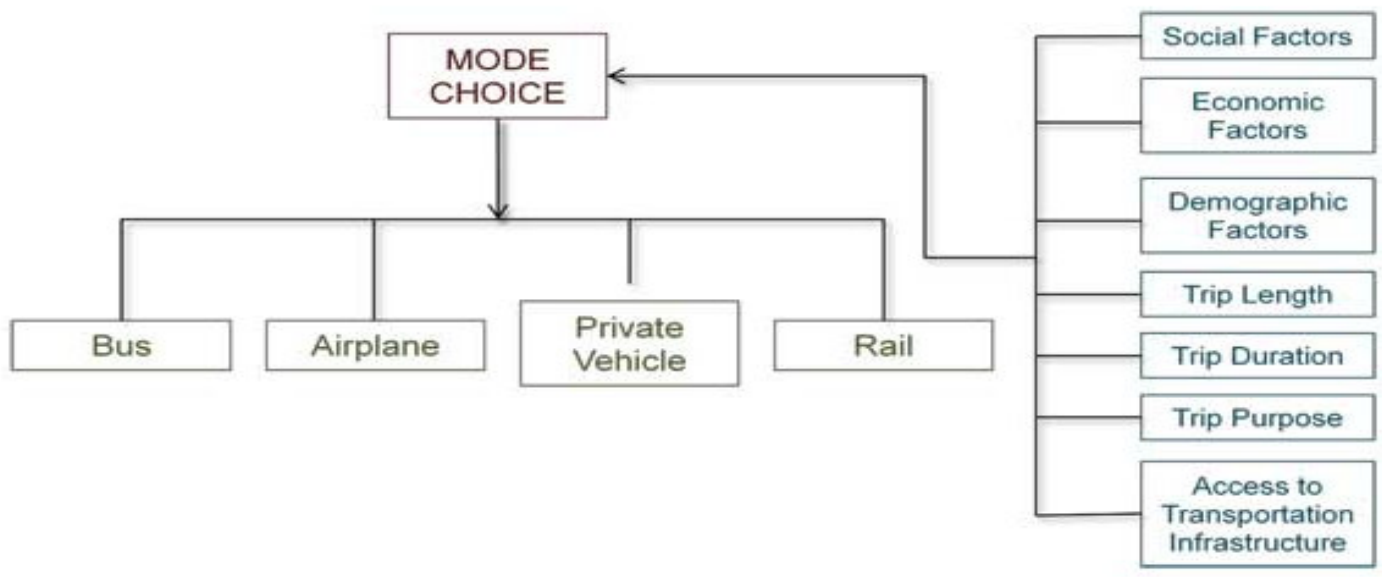

Source: (Rodrigue,2020) 
According to Rodrigue (2020), mode choice analysis is the third step in the conventional fourstep transportation forecasting model. The steps, in order, are trip generation, trip distribution, mode choice analysis, and route assignment. Trip distribution's zonal interchange analysis yields a set of origin destination tables that tells where the trips will be made. Mode choice analysis allows the modeller to determine what mode of transport will be used, and what modal share results. As shown on Figure 2.1 mode choice or determinants of transport choice is influenced by social, economic, demographic, trip length, trip duration, trip purpose and access to transport infrastructure Typically, these model estimate how many persons will ride public transit, how many will use private vehicles, how many will use the train and those who will prefer air transport. Further sophistication of this model may include identifying sub mode choice among different transit services and estimating the number of car pools or van pools of various sizes for high occupancy vehicle facilities. Mode choice models are found in numerous formulations, but the most common are based on the probabilities estimated by some variation or sophistication of the logit function. The common logit mode choice relationship states that the probability of choosing a particular mode for a given trip is based on the relative values of the costs and levels of service on the competing modes for the trip interchange being considered, (Rodrigue, 2020)

The level of service provided by a particular mode for a specific trip interchange is usually represented in part by the travel time for that interchange as computed from the transit and roadway networks. The travel time components used to represent level of service include the in-vehicle travel time for each mode and the out-ofvehicle time required to use that mode, such as walking to a transit stop or from a parking lot. The level of service also includes the waiting time likely to be experienced, either to board transit or to transfer. The delay due to roadway traffic congestion is included inherently by using attenuated speeds for congested roadway network links, (Ralph,2011)

The travel time and cost of a trip are usually combined using an estimate of the cost of time to convert either cost or time to the terms of the other. The cost of time is usually a variable, based on the economic level of the traveller. Although the mode choice model may be developed using the economic level of individual travellers, forecasts of mode choice are prepared for different economic groups, such as high, medium, and low income travellers. The resulting combination of time and cost is commonly referred to as the "utility" or "generalized cost."(Ralph,2011) and (Eboli \& Mazzulla, 2018).

\subsection{Passenger transport modal choice}

Mode choice behaviour for passenger transport is a key element in public transport planning, as it has a direct impact on the design of urban transport system structure, and is also the basis for urban public transport planning and management policymaking. Discrete choice models (DCMs) are a typical method of research on consumer choice behaviour originally applied in economics. Paying for using public transport, public transport users are actually consumers. Therefore, their mode choice behaviour is actually a consumer choice behaviour. Discrete choice models (DCMs) explain individual choice behaviour as the consequence of preferences that an individual makes with the assumption that the consumer chooses the most preferred option. Under certain assumptions, consumer preferences can be represented by a utility function in which the choice process somehow seems to be a sort of "black box." Its inputs are the attributes of available options and the individual's characteristics, and its output is the utility value of each alternative, (Ralph,2011)

The available transport modes from Harare to Gaborone includes flying which takes $4 \mathrm{~h} 8 \mathrm{~m}$ and costs usd

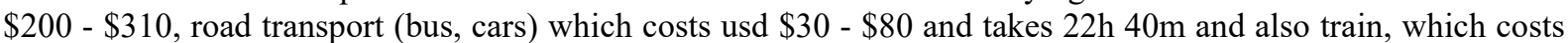
between usd $\$ 10-\$ 50$ and takes $27 \mathrm{~h} 5 \mathrm{~m}$. The total distance between Harare and Gaborone is $1068.9 \mathrm{~km}$ using road. These variables form the basis for determining which mode is best for travellers and passengers, (Mbara 2016)

\subsection{The socio-economic, travel characteristics and reasons for modal choice on Harare to Gaborone route} Urban structure is a framework of housing, employment and developments such as hospitals, education centres, and leisure facilities that combined with socioeconomic factors, influence and shape travel patterns. As such, varying urban structures in cities can be anticipated such low density, high density, urban sprawl and public transport resulting in differing travel patterns. Therefore, it emphasizes on land uses and job housing factors, and how they influence trip lengths, modes (Reilly, 2016). Also, it focuses on how different sections of society are taking up what mode of travel, depending on their various factors. Hence it focuses a lot on the socio-economic, and socio-demographics. Another major thing that has been completely underestimated is, while doing the planning for large or small cities, it is always taken for granted that that land over there is absolutely a flat terrain. Which is why, many such cases do not work, as the systems of travel vary from place to place in terms of topography, spatial patterns, and climate. Also, how the planning policies play a role in it,(Ralph, 2011). The distance between Harare and Gaborone being the same passengers chose different modes mainly based on the need for urgency, cost, comfort of the mode, boarder points restrictions, car ownership and sometimes ability to drive longer distances, (Munuhwa et al, 2020) 


\subsection{The relationship between socio-economic, travel characteristics and reasons for modal choice}

There are other socio demographic factors such as the composition of the mass like age, gender, income which play a significant role in the determination of modal choice on Harare to Gaborone route (Khayesi 2016). The major components like socioeconomics, demographics, topography, climate, culture, land use and how it affects the modal choice and their travel behaviour. Overall explanatory factors for determining travel behaviour can be broadly categorized as: socio-economic and demographic characteristics, spatial development patterns, policies directly or indirectly affecting travel behaviour, as well as national cultures or individual preferences (Khayesi 2016). In as so far as medium- and longer-distance travel has been analysed, the focus has been primarily on the impact of socioeconomic factors at the individual and household levels (Algers, 2013; Georggi and Pendyala, 2019; Mallett, 2019, OÕNeill and Brown, 2017). The relevance of the spatial configuration of land use and transport infrastructure for these trips has rarely been Socio economic factors are more critical to analyse to determine any kind of relationships on travel patterns. Socioeconomic characteristics consists of age, income, household size, and car ownership. Trip maker such as socio-demographic factors such as age, gender, ethnicity is included in travel because of two reasons-

One, because they may directly influence travel behaviour, and two, because, as proxies to more difficult to observe factors, such as preferences, tastes, choices, resource constraints and social conventions. Income and vehicle access are for example good indicators of an individual's access to resources, whereas, gender, age and ethnicity variables provide partial hints to individual's tastes and travel preferences (Daniels, 2012).

\section{Methodology}

\subsection{Research philosophy}

According to Saunders a research philosophy allows the researcher to think critically about the enhancement of knowledge (Saunders et al., 2013). This research study adopted interpretivism in order to gain a deeper understanding of determinants of passenger transport modal choice in Harare to Gaborone route.

\subsection{Research strategy}

This research study adopted quantitative research strategy because it sought to interpret how determinants of passenger transport modal choice was understood and implemented from Harare to Gaborone hence then quantitative research strategy can focus on the process of meaning constructing and clarify what and how meanings are embodied through an inductive logic as denoted by Creswell (2014).

\subsection{Research design}

According to Saunders (2010) a research design refers to the way the study is designed, that is, the method which is used to carry out a research. This research study adopted a cross sectional survey because this research is the investigation in which quantitative data is gathered and analysed in order to describe the specific phenomenon in its current trends. Cross sectional survey research design was chosen because it enabled the researcher to generalize the findings to a larger population of people in Harare, Zimbabwe.

\subsection{Sample size and sampling technique}

According to Kothari (2013) the sample size is an important feature of any empirical study in which the main goal is to make inferences about a population from a sample. In this research study the researcher adopted a sample size of 30 people. The researcher adopted 30 people as a sample through the use of Raosoft sample size calculator attained at http://www.raosoft.com. The researcher typed the website on an opera mini browser and the site requested the population size before determining the sample size of 30 people. In the survey part the male and female respondents had equal chances of taking part as random selection was used. Purposive sampling was adopted and seventy percent of the population of the study was used as a sample for the research in question. According to Borg and Gall (2013) there are two main types of sampling methods, which are probability sampling and non-probability sampling. For the purpose of the study non-probability sampling was adopted. Saunders (2013) and Cresswell (2012) noted that non probability sampling is a situation where there is no procedure to estimate the probability that each element has the chance of being selected. It involves the selection of elements based on assumptions regarding the population of interest, which forms the criteria for selection. In this research study nonprobability sampling methods such as convenience simple random sampling and purposive sampling were used.

\subsection{Instruments}

The data collection instruments that this study adopted was the use of questionnaires to gather data from research participants. The research instruments was used to gather primary data from the field of study. It was conducted in Harare to obtain the information needed to conduct the study. Cresswell (2013) defined questionnaires as prepared set of questions which are used to obtain information from a respondent of the study. Open-ended questions were utilized because they allows a respondent to answer questions in their own words whiles close- 
ended questions specified all the possible answers and respondent make choice among them. Questionnaires were selected because they provide answers that were easier to interpret and tabulate. The questionnaires designed for this study basically contained closed ended questions with responses provided for the respondent to choose. This was used to obtain reaction to determinants of passenger transport modal choice.

\subsection{Data collection procedures}

Questionnaires were distributed to the respondents and then they were collected after a few hours giving the respondents ample time to complete the questionnaires. The researcher was booked for an appointment for carrying out interviews on the day and that they introduced themselves letting the authorities of their intentions to carry out their academic survey. The researcher managed to carry out only a handful of interviews with the marketing executives because the other respondents were not available for the interviews and or had other commitments. The respondents were selected using the judgmental sampling technique to save on time. Additionally, the researcher played their part, by working within the agreed time frame with the respondents, a period that would not affect their operations. The researcher also allowed respondents to respond in the manner they please. The researcher did not influence the respondents in anyway, be it be offering monetary incentives or by any other form of cohesion. The researcher did not alter the findings or their responses. Once the data was collected, the researcher analysed it as it was.

\subsection{Data analysis}

The researcher summarized and measured the data using the Statistical Package for Social Sciences (SPSS software). The research then compared the dependent and independent variables for data analysis using variable mean. The researcher used names which comprises of grouping variables for descriptive statistics. A lot of information was gathered so in this chapter the researcher focused on matching data collected with the research objectives and questions. Data was presented and analysed using Pie charts, Bar graphs, or other relevant data presentation methods. The researcher designed different codes that were in line with data collected so that the researcher would be able to enter data and analyse it accordingly for example on gender male (1) and female (2).

\section{Results}

\subsection{Response rate}

A total of 30 questionnaires were prepared for this research study and 30 were returned. This represented a response rate of $100 \%$ which was adequate for data analysis and conforms to previous researches such as (Mazikana, 2018; Desbordes, 2016, Sifile et al., 2018 and Makanyeza, 2015) who stipulated that a response rate of $70 \%$ and above is deemed adequate and excellent for a research study.

\subsection{Gender}

Respondents of this study were asked to indicate their gender and their responses were captured down in table 5.1 below

Table 4.1: Gender analysis

\begin{tabular}{|l|l|l|}
\hline & Frequency & Percent \\
\hline Male & 18 & 60.0 \\
Female & 12 & 40.0 \\
Total & 30 & 100.0 \\
\hline
\end{tabular}

Source: SPSS output

According to the survey which was undertaken in this research study, $60 \%$ of the responses were male staff whereas $30 \%$ were females. This shows that the population is fairly balanced but the selected respondents' panel had more male staff than females. In a similar study Makanyeza (2015) noted that there are many males than females in Zimbabwe. ZIMSTAT (2012a) in Makanyeza (2015) noted that there are more males (51.94\%) than females (48.06\%) in Zimbabwe. As a result, the findings which were obtained in above table 5.1 above were highly expected

\subsection{Age group}

Respondents of this study were asked to indicate their age group and their responses were captured down in table 5.2 below 
Table 4.2: Age group of respondents

\begin{tabular}{|l|l|l|}
\hline & Frequency & Percent \\
\hline $18-25$ & 12 & 40.0 \\
$26-35$ & 6 & 20.0 \\
$37-47$ & 9 & 30.0 \\
47 and above & 3 & 10.0 \\
Total & 30 & 100.0 \\
\hline
\end{tabular}

Source: SPSS output

Table 4.2 above shows that $12(40 \%)$ of the respondents were in the age range of $18-25$ years, $6(20 \%)$ were in the age range of 26-35 years, $9(30 \%)$ were in the age range of $37-47$ years whilst $3(10 \%)$ were in the age range of 47 years and above. From the data obtained in this research study it can be inferred that the majority of the respondents were in the age range of 18-25 years. In a similar study conducted by Mazikana (2018) it was noted that there appears to be a fine balance in the age group with more youthful staff hence the results attained in this research study were highly expected. Meanwhile it can also be concluded that the results attained in this research study were reliable as the majority of the respondents were above 18 years, this age group provides honest answers (Makanyeza, 2014).

\subsection{Highest level of education}

Respondents of this research study were asked to indicate their highest level of education and their responses were captured down in figure 4.1 below.

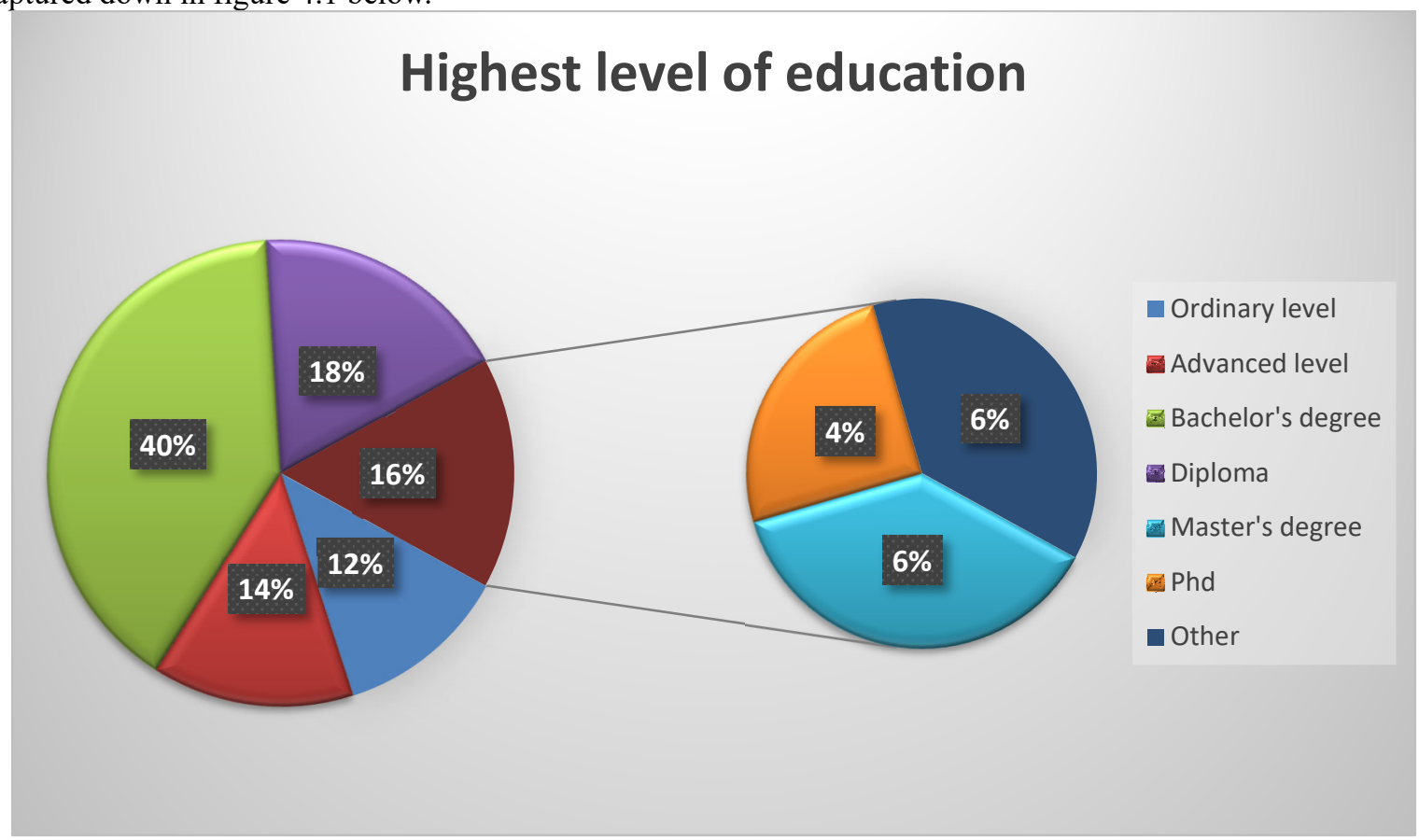

Figure 4.1: Highest level of education

Source: Survey conducted for this study

Figure 4.1 above shows one respondent had a Doctoral degree $1(4 \%)$ and Master's degree $2(6 \%)$ while a considerable number of the respondents have attained a Bachelor's degree 12 (40\%) and a Diploma $5(18 \%)$. More so, few respondents had attained advanced level education $4(14 \%)$, ordinary level education $3(12 \%)$, other certificates such as Zimbabwe Junior Certificate education and Grade 7 education were 2 (6\%). This implies that the study sample mainly consists of educated people. This can be attributed to the general observation that the respondents who were more responsive to participate in the survey were those who appreciated the passenger transport modal choice research perhaps because they would have done research elsewhere especially at the tertiary education level. More over from the figure 4.1 above it can be denoted that the respondents had the capacity to answer questions in the questionnaire. Mazikana (2018) also reckons that knowledge is needed. This is in support with the view that most of the respondents who completed the questionnaire had bachelor's degree and they were able to interpret the questions about passenger transport modal choice in Harare, Zimbabwe.

\subsection{Reliability test results}

To determine the reliability of the data collection instrument which the questionnaire on determinants of passenger 
transport modal choice. A case of Harare to Gaborone route, the research study subjected to a pilot study. The Cronbach's Alpha measure of internal consistency was then adopted to determine the reliability of the questionnaires of the research study on determinants of passenger transport modal choice. The alpha measures internal consistency by establishing whether certain items measure the intended variable. Nunnaly (2017) established that the Alpha value threshold at 0.7 which the study benchmarked against. Table 4.3 below presents the reliability test results.

Table 4.3: reliability of the study construct

\begin{tabular}{|l|l|l|}
\hline Construct & Number of items & Cronbach's $\alpha$ \\
\hline Speed & 30 & 0.897 \\
\hline Appearance of vehicle & 29 & 0.786 \\
\hline Safety & 29 & 0.820 \\
\hline Transfer fare(Cost) & 29 & 0.826 \\
\hline Travel distance comfort & 29 & 0.877 \\
\hline Waiting time at bus stop & 30 & 0.782 \\
\hline
\end{tabular}

The results attained in table 4.3 above shows that all the Cronbach's values were above 0.75 indicating that the items measured single unidimensional latent constructs. The results are in line with the sentiments of Tavakol and Dennick (2015) who noted that the accepted range of Cronbach's alpha is between 0.70 and 0.90 and such research is deemed reliable. The Coefficients Alpha Coefficients in table 4.2 reveal that all the scales were significant, having an alpha above the prescribed threshold of 0.7 . The highest level of validity was recorded in speed of the transport vehicle $(\alpha=0.897)$ followed by Travel distance comfort $(\alpha=0.877)$. The study thus found that the analysis was valid and could be used for further analysis.

\section{Conclusions and policy implications}

From the study it can be concluded that the most preferred mode of transport from Harare to Gaborone is road and specifically the bus means of transport. Passengers choose the mode of transport influenced by the following determinants; total travel time, speed of the transport mode, urgency of passengers, passenger safety issues, customer service provided by the operator ( including assisting passengers in clearing of goods and informing them of foreign country requirements), cost of travelling, physical appearance of the transport vehicle, waiting times at terminal points, income levels or availability of substitute modes of transport going the same route. Commuters' choice of commercial vehicles generally decreases with an increase in transport fare. A similar observation is reported by Eboli and Mazzulla (2018) for bus users in Cosenza, Italy. However, generally, there is difference in the choice of commercial vehicle by trip purposes that is commuting and noncommitting trips. Confirming the observation by Foote et al. (2011) that the quality of each of the public transport attributes is related to the importance each passenger places on it. The findings of this study may be used by transport operators and policy-makers to formulate strategies for the improvement of public transport to attract private car users in order to resort to the use of public transport; this will help reduce road congestion or traffic situation in urban areas like Harare and Gaborone.

\section{References}

Adamowicz, W. L., Louviere, J., \& Williams, M. (2014). Combining stated and revealed preference methods for valuing environmental amenities. Journal of Environmental Economics and Management 26, 271-292.

Adamowicz, W., Louviere, J., \& Swait, J. (2018). Introduction to Attribute-Based Stated Choice Methods. Washinton, U.S.A: Resource Valuation Branch Damage Assessment Center, NOAA, U.S Department of Commerce.

Afful, D. (2011). The Challenges Confronting Private Bus Operating in Ghana. Netherland: Unpublished MBA Thesis.

Alpizar, F., \& Carlsson, F. (2011). Policy Implications and Analysis of the Determinants of Travel Mode Choice. An Application of Choice Experiments to Metropolitan Costa Rica. Costa Rica: Working Paper in Economics no. 5, Department of Economics, Göteborg University.

Carlsson, F., Frykblom, P., \& Liljenstolpe, C. (2013). Valuing wetland attributes: An application of choice experiments. Ecological Economics 47, 95-103.

Catalano, M., Lo Casto, B., \& Migliore, M. (2018). Car sharing demand estimation and urban transport demand modelling using stated preference techniques. European Transport, 33-50.

Eboli, L \& Mazzulla, G. (2018). A stated preference experiment for measuring service quality in public transport. Transportation Planning and Technology 31:5, 509-523.

Foote, J., Stuart, G., \& Elmore-Yalch, R. (2011). Exploring customer loyalty as a transit performance measure. Transportation Research Record 1753, p. 93-101.

Hensher, D. A. (2014). Stated preference analysis of travel choices: The state of practice. Transportation 21(2), 107-133. 
Hensher, D. A. (2011). The valuation of commuter travel time savings for car drivers in New Zealand: Evaluating alternative model specifications. Transportation 28, 101-118.

Hensher, D. A., \& Greene, W. H. (2001). The mixed logit model: The state of practice and warnings for the unwary. Working Paper, School of Business, The University of Sidney.

http://www.dailynews.gov.bw/news-details.php?nid=5875 accessed on 22 March 2020

http://www.raosoft.com

https://transportgeography.org/?page_id=1731 accessed on the $21^{\text {st }}$ of March 2020

Jean-Paul Rodrigue (2020), The Geography of Transport Systems, fifth edition, New York: Routledge, 456 pages. ISBN 978-0-367-36463-2

Mbara T,C (2015) Achieving Sustainable Urban Transport in Harare, Zimbabwe: What are the Requirements to Reach the Milestone? University of Johannesburg,, Journal of Transport and Supply Chain Management.

Ralph, B. (2011), Determinants of Transport Mode Choice: A Comparison of Germany and the USA

Journal of Transport Geography, DO - 10.1016/j.jtrangeo.2010.07.005

Munuhwa Shakerod, et al. (2020), Approaches for Reducing Urban Traffic Congestion in The City of Harare, Journal of Economics and Sustainable Development, ISSN2222-1700(Paper) ISSN)2222-2855(Online) 